\title{
Fermi-Dirac corrections to the relic abundances
}

\author{
A.D. Dolgov ${ }^{a}$ and K. Kainulainen ${ }^{b}$ \\ ${ }^{a}$ Department of Physics, University of Michigan, Ann Arbor, MI 48109, USA \\ ITEP, 117259 Moscow, Russian Federation \\ ${ }^{b}$ Theoretical Physics Institute, University of Minneapolis, Minneapolis, MN 55455, USA
}

Received 17 November 1992

Accepted for publication 5 April 1993

\begin{abstract}
We derive an equation for the evolution of the number density of a massive particle species in the early universe, which correctly accounts for the Fermi-Dirac (FD) statistics. The FD-corrections are sizable and potentially important if the decoupling from the thermal equilibrium takes place at temperatures of the order of, or less than the mass of the particle. This is the case e.g. for a few MeV tau neutrino with the ordinary weak interactions.
\end{abstract}

\section{Introduction}

Calculation of the evolution of the number densities of new hypothetical massive particles has become a very important part of the study of the early universe. A lot of work has been devoted to the calculation of relic abundances of various cold dark matter candidates in the present day universe [1-5]. It is usually correct to assume that the particles remain in thermal equilibrium to temperatures much below their mass, so that around the decoupling temperature $T_{\mathrm{d}}$ it is adequate to approximate the Fermi-Dirac (FD) statistics with the Maxwell-Boltzmann (MB) statistics. One can then show that in these temperatures $(T \ll m)$ the number density $n$ is governed by the equation $[1,6-9]$

$$
\frac{\mathrm{d} n}{\mathrm{~d} t}+3 H n=\left\langle v_{\mathrm{M} \phi 1} \sigma\right\rangle\left(n_{\mathrm{eq}}^{2}-n^{2}\right),
$$

where $n_{\text {eq }}$ is the equilibrium density and $H=\left(\frac{4}{45} \pi^{3} g(T) T^{4} / M_{\mathrm{Pl}}^{2}\right)^{1 / 2}$ is the Hubble expansion factor where $M_{\mathrm{Pl}}$ is the Planck mass and the function $g(T)$ is related to the total energy density $\rho$ by $\rho(T) \equiv \frac{1}{30} \pi^{2} g(T) T^{4} .\left\langle v_{\mathrm{M} \phi 1} \sigma\right\rangle$ is a thermally averaged cross section to be defined precisely below; here it suffices to note that it is independent of $n$. This separation of the RHS of eq. (1) into a power function of $n$ multiplied by a $t$-dependent factor can only be obtained in the Maxwell-Boltzmann approximation. 
If the decoupling temperature is of the order of the mass, it is not correct to use the MB-statistics and hence eq. (1) is no more adequate. One potentially interesting physical candidate for which $T_{\mathrm{d}} \sim m$ is a few $\mathrm{MeV}$ tau neutrino. A tau neutrino in this mass range would have a significant effect on the nucleosynthesis [10].

In general it is not possible to write down an equation for the number density $n$ directly, but as we shall show below, a tractable equation can be found for the so-called pseudo-chemical potential $z(t)$ [7,11], that is of the functional form

$$
\frac{\mathrm{d} z}{\mathrm{~d} t}=F(z(t), t)
$$

Knowledge of $z(t)$ allows the calculation of all thermodynamical quantities as simple integrals over momentum at each time $t$.

In sect. 2 we will derive the explicit form of eq. (2). In sect. 3 we will derive the MB-limit for that equation and compare our result to the literature. In sect. 4 we solve our equations numerically in a simple example and compare the solution of eq. (2) with the correct statistics to the numerical as well as to one often used approximative solution of eq. (1). Sect. 5 contains our conclusions and discussion.

\section{The evolution equation}

We will derive the evolution equation for fermions (called neutrinos in what follows) under the standard assumption made in the literature that all the helicity states are equally populated *. Our starting point is then the Boltzmann equation for the scalar distribution function $f(p, t)$ in the flat Friedmann-RobertsonWalker space-time $[7,13]$,

$$
E\left(\partial_{t}+p H \partial_{p}\right) f(p, t)=C_{\mathrm{E}}(p, t)+C_{\mathrm{I}}(p, t)
$$

where $E=\left(\mathrm{p}^{2}+\mathrm{m}^{2}\right)^{1 / 2}$ and $C_{\mathrm{E}}(p, t)$ and $C_{\mathrm{I}}(p, t)$ are the elastic and inelastic collision integrals respectively. Elastic collisions are responsible for maintaining the kinetic equilibrium, but the exact form of the elastic collision integral is not important for our present purposes. The inelastic collision integral on the other hand is given by

$$
C_{\mathrm{I}}\left(p_{1}, t\right)=\frac{1}{2} \sum_{n} \int \prod_{i=2}^{4} \frac{\mathrm{d}^{3} p_{i}}{(\pi)^{3} 2 E_{i}}(2 \pi)^{4} \delta^{4}\left(p_{1}+p_{2}-p_{3}-p_{4}\right)
$$

\footnotetext{
* Note that this is not necessarily true in a realistic case, where the interactions may be chiral. This
} problem is considered elsewhere [12]. 


$$
\begin{aligned}
& \times \sum_{s_{i}, i=2}^{4}\left[\left|\mathscr{M}_{34 \rightarrow 12}^{(n)}\right|^{2} f_{n}\left(p_{3}, t\right) \bar{f}_{n}\left(p_{4}, t\right)\left(1-f\left(p_{1}, t\right)\right)\left(1-\bar{f}\left(p_{2}, t\right)\right)\right. \\
& \left.-\left|\mathscr{M}_{12 \rightarrow 34}^{(n)}\right|^{2} f\left(p_{1}, t\right) \bar{f}\left(p_{2}, t\right)\left(1-f_{n}\left(p_{3}, t\right)\right)\left(1-\bar{f}_{n}\left(p_{4}, t\right)\right)\right],
\end{aligned}
$$

where the sum over $n$ includes all the particles in the system that the neutrinos interact with and the sum over $s_{i}$ goes over the spins. Note the normalization factor $\frac{1}{2}$ in front of the integral, which is sometimes forgotten in the literature. The overbars above the distribution functions refer to antiparticles. In what follows we will assume vanishing chemical potentials so that for all the particles $\bar{f}=f$.

In the usual approach leading to eq. (1), the following assumptions are made:

(i) The neutrinos are in kinetic equilibrium.

(ii) Particles involved in the sum over $n$ in eq. (4) are in complete thermal equilibrium.

(iii) MB-statistics is adequate for neutrinos.

Here we will also make the assumptions (i) and (ii), but we will relax the assumption (iii). Due to (i) and (ii) we can write the distribution functions as

$$
\begin{gathered}
f(p, t) \rightarrow f(p, z) \equiv\left(\mathrm{e}^{\beta E+z}+1\right)^{-1}, \\
f_{n}(p, t) \rightarrow f_{n}(p) \equiv\left(\mathrm{e}^{\beta E_{n}}+1\right)^{-1},
\end{gathered}
$$

where $\beta \equiv 1 / T$ and $z(t)$ is the pseudo-chemical potential [7,11]. It should be noted that, in contrast to the usual chemical potentials in equilibrium, $z(t)$ appears with the same sign in both the distributions for particles and antiparticles. Inserting (5a) and (5b) into (4) and by using unitarity and CPT or the principle of detailed balance one can integrate the RHS of eq. (3) and get

$$
\begin{aligned}
S_{\mathrm{I}}(z) \equiv & \sum_{\text {spin }} \int \frac{\mathrm{d}^{3} \bar{p}}{(2 \pi)^{3}} \frac{1}{E} C_{\mathrm{I}}(p, t) \\
= & \left(\mathrm{e}^{2 z}-1\right) \sum_{n} \int \prod_{i=1}^{4} \frac{\mathrm{d}^{3} p_{i}}{(2 \pi)^{3} 2 E_{i}}(2 \pi)^{4} \delta^{4}\left(p_{1}+p_{2}-p_{3}-p_{4}\right) \\
& \times \sum_{s_{i}, i=1}^{4}\left|\mathscr{M}_{12 \rightarrow 34}^{(n)}\right|^{2} f\left(p_{1}, z\right) f\left(p_{2}, z\right)\left(1-f_{n}\left(p_{3}\right)\right)\left(1-f_{n}\left(p_{4}\right)\right) .
\end{aligned}
$$

Of course, a similar integral over the elastic collision term $C_{\mathrm{E}}(p, t)$ vanishes under the conditions (i) and (ii). Performing the same operation to the LHS of eq. (3), we find

$$
\sum_{\text {spin }} \int \frac{\mathrm{d}^{3} p}{(2 \pi)^{3}}\left(\partial_{t}-p H \partial_{p}\right) f(p, t)=-A(z) \frac{\mathrm{d} z}{\mathrm{~d} t}+B(z)
$$


where the functions $A(z)$ and $B(z)$ are defined as

$$
\begin{aligned}
& A(z) \equiv g \int \frac{\mathrm{d}^{3} p}{(2 \pi)^{3}} f(p, z)^{2} \mathrm{e}^{\beta E+z} \\
& B(z) \equiv g \int \frac{\mathrm{d}^{3} p}{(2 \pi)^{3}}\left[\left(H+\frac{1}{T} \frac{\mathrm{d} T}{\mathrm{~d} t}\right) \frac{E}{T}-H \frac{m^{2}}{E T}\right] f(p, z)^{2} \mathrm{e}^{\beta E+z},
\end{aligned}
$$

where $g$ is the neutrino spin factor. Changing the variable from $t$ to $x \equiv m / T$ and inserting (6), (7) into (3), we obtain the equation for $z(x)$ of the form (2),

$$
\frac{\mathrm{d} z}{\mathrm{~d} x}=\left(A(z) \frac{x}{T} \frac{\mathrm{d} T}{\mathrm{~d} t}\right)^{-1}\left(-B(z)+S_{\mathrm{I}}(z)\right) .
$$

The function $B(z)$ on the RHS of eq. (9) represents the "free" part due to the expansion of the universe, and the function $S_{\mathrm{I}}(z)$ is the interaction term. The remaining part of this section is involved in writing (9) in a more explicit way.

Let us define the functions $J_{n}(x, z)$ as

$$
J_{n}(x, z) \equiv \int_{1}^{\infty} \mathrm{d} y y^{n}\left(y^{2}-1\right)^{1 / 2} \frac{\mathrm{e}^{x y}}{\left(1+\mathrm{e}^{x y+z}\right)^{2}} .
$$

With help of these integrals we can write the functions $A(z)$ and $B(z)$ as

$$
\begin{aligned}
& A(z)=\frac{g}{2 \pi^{2}} m^{3} \mathrm{e}^{z} J_{1}(x, z) \\
& B(z)=\frac{g}{2 \pi^{2}} m^{3} \mathrm{e}^{z} x\left[\left(H+\frac{1}{T} \frac{\mathrm{d} T}{\mathrm{~d} t}\right) J_{2}(x, z)-H J_{0}(x, z)\right] .
\end{aligned}
$$

The collision integral $S_{\mathrm{I}}(z)$ can always be reduced to five dimensions. We will choose our independent variables as the three energies $E_{1}, E_{2}$ and $E_{3}$ and the two angles, $\theta$, the angle between the incoming particles and $\phi$, the (acoplanarity) angle between the planes of the incoming and outgoing particles. With this choice of parameters one obtains, after elementary albeit somewhat lengthy manipulations, the result

$$
\begin{aligned}
S_{\mathrm{I}}(z)= & \frac{m^{4}}{512 \pi^{6}}\left(\mathrm{e}^{2 z}-1\right) \\
& \times \sum_{n} \int \mathscr{D} \Phi \int_{0}^{2 \pi} \mathrm{d} \phi \sum_{\text {spin }}\left|\mathscr{K}_{\mathrm{I}}^{(n)}(u, v, t, \cos \theta, \phi)\right|^{2},
\end{aligned}
$$


where

$$
\begin{aligned}
\int \mathscr{D} \Phi \equiv & \int_{1}^{\infty} \mathrm{d} u \int_{1}^{\infty} \mathrm{d} v \int_{-1}^{1} \mathrm{~d} \cos \theta \frac{\mathscr{P}_{u} \mathscr{P}_{v}}{\kappa(u, v, \theta)} \int_{t_{-}}^{t_{+}} \mathrm{d} t \mathrm{e}^{x u+x v} \\
& \times\left(\mathrm{e}^{x u+z}-1\right)^{-1}\left(\mathrm{e}^{x v+z}+1\right)^{-1}\left(\mathrm{e}^{x t}+1\right)^{-1}\left(\mathrm{e}^{x(u+v-t)}+1\right)^{-1},
\end{aligned}
$$

where $\mathscr{P}_{\alpha} \equiv\left(\alpha^{2}-1\right)^{1 / 2}$ and $\kappa(u, v, \theta) \equiv\left|\mathbf{p}_{1}+\mathbf{p}_{2}\right| / m=\left(\mathscr{P}_{u}^{2}+\mathscr{P}_{v}^{2}-\right.$ $\left.2 \mathscr{P}_{u} \mathscr{P}_{v} \cos \theta\right)^{1 / 2}$ and we have used the scaled variables $u \equiv E_{1} / m, v \equiv E_{2} / m$ and $t \equiv E_{3} / m$. The $t$-integration limits are given by $t_{ \pm}=\frac{1}{2}(u+v \pm$ $\left.\kappa(u, v, t) \sqrt{1-4 m_{n}^{2} / s}\right)$, where $m_{n}$ is the mass of the species $n$ and $s$ is the usual invariant $s \equiv\left(p_{1}+p_{2}\right)^{2}=2 m^{2}\left(u v+1-\mathscr{P}_{u} \mathscr{P}_{v} \cos \theta\right)$. In some cases the matrix element $\mathscr{M}$ may be simple enough to allow further integrations in (12), but due to the FD-distribution functions a complete analytic evaluation is not possible even in the simplest case of a constant matrix element. In particular in the simple though not very realistic case of an $s$-dependent matrix element squared (12) can be integrated down to two dimensions.

Finally, in order to fix the time-temperature relation we will assume that the universe expands adiabatically. Then

$$
\frac{1}{T} \frac{\mathrm{d} T}{\mathrm{~d} t}=-H /\left(1+\frac{1}{3} \frac{T}{h} \frac{\mathrm{d} h}{\mathrm{~d} T}\right),
$$

where the function $h(T)$ is related to the entropy density of the interacting species $s$ by $s(T) \equiv \frac{2}{45} \pi^{2} h(T) T^{3}$.

Altogether we can now write eq. (9) into a more explicit form,

$$
\begin{aligned}
\frac{\mathrm{d} z}{\mathrm{~d} x}= & -\frac{J_{0}(x, z)}{J_{1}(x, z)}+\frac{1}{3} \frac{T}{h} \frac{\mathrm{d} h}{\mathrm{~d} T} \frac{J_{2}(x, z)-J_{0}(x, z)}{J_{1}(x, z)} \\
& +\sinh z \gamma(T) \frac{g x M_{\mathrm{Pl}}}{128 \pi^{4} m J_{1}(x, z)} \\
& \times \sum_{n} \int \mathscr{D} \Phi \int_{0}^{2 \pi} \mathrm{d} \phi \frac{1}{g^{2}} \sum_{\text {spin }}\left|\mathscr{M}_{\mathrm{I}}^{(n)}(u, v, t, \theta, \phi)\right|^{2},
\end{aligned}
$$

where

$$
\gamma(T) \equiv\left(\frac{4}{45} \pi^{3} g(T)\right)^{-1 / 2}\left(1+\frac{1}{3} \frac{T}{h} \frac{\mathrm{d} h}{\mathrm{~d} T}\right)
$$

This is the main result of this section. Eq. (15) is actually simpler than what it looks; it is only the matrix element that needs to be calculated separately for each 
different case. Of course (15) can only be solved numerically, but there, even the multidimensional integrations are usually rather easy and the equation itself is well behaving.

If $z(x)$ is known one can obtain various thermodynamical quantities as simple integrals over the momentum,

$$
\alpha(x) \equiv \frac{g}{2 \pi^{2}} \int_{0}^{\infty} \mathrm{d} y y^{2} \bar{\alpha}(T, x)\left\{\exp \left[\left(y^{2}+x^{2}\right)^{1 / 2}+z\right]+1\right\}^{-1},
$$

where e.g. $\bar{\alpha}=T^{3}$ and $\bar{\alpha}=\left(y^{2}+x^{2}\right)^{1 / 2} T^{4}$ for the number and the energy densities respectively.

\section{The Maxwell-Boltzmann limit}

We will now derive the Maxwell-Boltzmann limit of eq. (15). In the MB-limit the distribution function for neutrinos $(5 \mathrm{a})$ is approximated by

$$
f(p, z)_{\mathrm{MB}}=\mathrm{e}^{-\beta E-z} .
$$

With this simplification the functions $J_{i}(x, z)$ can be expressed in terms of the modified Bessel functions of the second kind:

$$
\begin{aligned}
& J_{i}(x, z) \rightarrow \mathrm{e}^{-2 z} \frac{1}{x} K_{i+1}(x), \quad i=0,1 \\
& J_{2}(x, z) \rightarrow \mathrm{e}^{-2 z} \frac{1}{x}\left(\frac{3}{x^{2}} K_{2}(x)+\frac{1}{x} K_{1}(x)\right) .
\end{aligned}
$$

Secondly, the collision integral $S_{\mathrm{I}}(z)$ simplifies considerably. From (6) one obtains

$$
\begin{aligned}
S_{\mathrm{I}}(z)_{\mathrm{MB}}= & \left(1-\mathrm{e}^{-2 z}\right) g^{2} \int \prod_{i=1}^{2} \frac{\mathrm{d}^{3} p_{i}}{(2 \pi)^{3} 2 E_{i}} \exp \left[-\beta\left(E_{1}+E_{2}\right)\right] \\
& \times \sum_{n} \int \prod_{i=3}^{4} \frac{\mathrm{d}^{3} p_{i}}{(2 \pi)^{2} 2 E_{i}}(2 \pi)^{2} \delta^{4}\left(p_{1}+p_{2}-p_{3}-p_{4}\right) \frac{1}{g^{2}} \sum_{\text {spin }}\left|\mathscr{M}_{\mathrm{I}}^{(n)}\right|^{2} .
\end{aligned}
$$

The second line in (19) represents a Lorentz invariant quantity, namely the spin averaged cross section $\sigma^{(n)}(s)$ times the flux $F \equiv 4\left(\left(p_{1} \cdot p_{2}\right)^{2}-m^{4}\right)^{1 / 2} \equiv 4 E_{1} E_{2} v_{\mathrm{M} \emptyset 1}$, where the flux-related velocity factor $v_{M ø 1}$ is called the Møller velocity $[9,13,14]$. Thus

$$
S_{\mathrm{I}}(z)_{\mathrm{MB}}=\left(1-\mathrm{e}^{-2 z}\right) n_{\mathrm{eq}}^{2}(x) \sum_{n}\left\langle n_{\mathrm{M} \phi 1} \sigma^{(n)}\right\rangle
$$


where

$$
n_{\mathrm{eq}} \equiv g \int \frac{\mathrm{d}^{3} p}{(2 \pi)^{3}} \mathrm{e}^{-\beta E}=g \frac{T m^{2}}{2 \pi^{2}} K_{2}\left(\frac{m}{T}\right)
$$

and the averaged cross section $\left\langle v_{\mathrm{M} \varnothing \mathrm{l}} \sigma^{(n)}\right\rangle$ is defined as

$$
\left\langle v_{\mathrm{M} \phi 1} \sigma^{(n)}\right\rangle \equiv \frac{4 \pi^{4}}{m^{4} T^{2} K_{2}^{2}(m / T)} \int \frac{\mathrm{d}^{3} p_{1}}{(2 \pi)^{3}} \int \frac{\mathrm{d}^{3} p_{2}}{(2 \pi)^{3}} \mathrm{e}^{-\beta\left(E_{1}+E_{2}\right)} v_{\mathrm{M} \varnothing 1} \sigma^{(n)}(s) .
$$

It is easy to reduce $\left\langle v_{\mathrm{M} \varnothing 1} \sigma^{(n)}\right\rangle$ into an one-dimensional integral over $s$. We borrow the result of such an integration from the paper by Gondolo and Gelmini [9],

$$
\left\langle v_{\mathrm{M} \varnothing \mathrm{l}} \sigma^{(n)}\right\rangle=\frac{1}{8 m^{4} T K_{2}^{2}(m / T)} \int_{4 m^{2}}^{\infty} \mathrm{d} s \sqrt{s}\left(s-4 m^{2}\right) K_{1}\left(\frac{\sqrt{s}}{T}\right) \sigma_{\mathrm{CM}}^{(n)}(s) .
$$

Using eqs. (12), (18) and (20), eq. (9) can be written in the MB-limit as

$$
\frac{\mathrm{d} z}{\mathrm{~d} x}=-\frac{K_{1}(x)}{K_{2}(x)}+\frac{1}{x} \frac{T}{h} \frac{\mathrm{d} h}{\mathrm{~d} T}+\sinh z \frac{g}{\pi^{2}} \gamma(T) m M_{\mathrm{Pl}} K_{2}(x) \sum_{n}\left\langle v_{\mathrm{M} \phi 1} \sigma^{(n)}\right\rangle,
$$

where the function $\gamma(T)$ is as defined below eq. (15). Of course, in the MB-limit we can write (22) directly in terms of the number density $n$. First note that the RHS of eq. (7) can always be written as $\mathrm{d} n / \mathrm{d} t+3 H n$ and then use $n \equiv n(T, z)_{\mathrm{MB}}$ $=\mathrm{e}^{-z} n_{\mathrm{eq}}(T)$ in the expression (20). Then from (3), (7) and (20) one obtains the familiar result

$$
\frac{\mathrm{d} n}{\mathrm{~d} t}+3 H n=\sum_{k}\left\langle v_{\mathrm{M} \phi 1} \sigma^{(k)}\right\rangle\left(n_{\mathrm{eq}}^{2}-n^{2}\right)
$$

\section{The constant matrix element case}

We now wish to study quantitatively the size of the corrections arising from the use of the FD-statistics. To this end we consider the simplest case of a constant matrix element interaction and furthermore, we will neglect the entropy production, so that $\mathrm{d} h / \mathrm{d} T \equiv 0$. In order to mimic the strength of the standard model weak interactions we choose to parametrize the matrix element as $\left|\mathscr{M}_{\mathrm{I}}\right|^{2}=$ $\alpha G_{\mathrm{F}}^{2} m^{4}$, where $\alpha$ is a free parameter. A value $\alpha \simeq 20$ would correspond to the interaction strength of an ordinary neutrino in the early universe at a few $\mathrm{MeV}$ 
temperature. Accordingly, we will fix $g(T)=10.75$ corresponding to $T \simeq \mathrm{O}(1-100)$ $\mathrm{MeV}$. We then obtain from (15) a simplified equation,

$$
\frac{\mathrm{d} z}{\mathrm{~d} x}=-\frac{J_{0}(x, z)}{J_{1}(x, z)}-3.08 \times 10^{-4} \alpha\left(\frac{m}{\mathrm{MeV}}\right)^{3} \sinh z \frac{x}{J_{1}(x, z)} \int \mathscr{D} \Phi
$$

where, among other things, the trivial $\phi$-integration has been performed. As an aside let us note that in this case, and in fact in the more general case where $\left|\mathscr{M}_{1}\right|^{2}$ depends only on $s=\left(p_{1}+p_{2}\right)^{2}$, the integral over $\int \mathscr{D} \Phi$ can be reduced to a two-dimensional one,

$$
\begin{aligned}
\int \mathscr{D} \Phi\left|\mathscr{M}_{\mathrm{I}}\right|^{2}(s)= & 4 \pi \sum_{n} \sum_{\text {spin }} \int_{2 x}^{\infty} \mathrm{d} p \int_{0}^{\left(p^{2}-4 x^{2}\right)^{1 / 2}} \mathrm{~d} q \mathrm{e}^{-p}\left|\mathscr{M}_{\mathrm{I}}\right|^{2}(s) \\
& \times \ln \left(\frac{\underline{\cosh } \frac{1}{4}(p+q)}{\cosh \frac{1}{4}(p-q)}\right) \ln \left(\frac{\cosh \left(\frac{1}{4}(p+q V)+\frac{1}{2} z\right)}{\cosh \left(\frac{1}{4}(p-q V)+\frac{1}{2} z\right)}\right),
\end{aligned}
$$

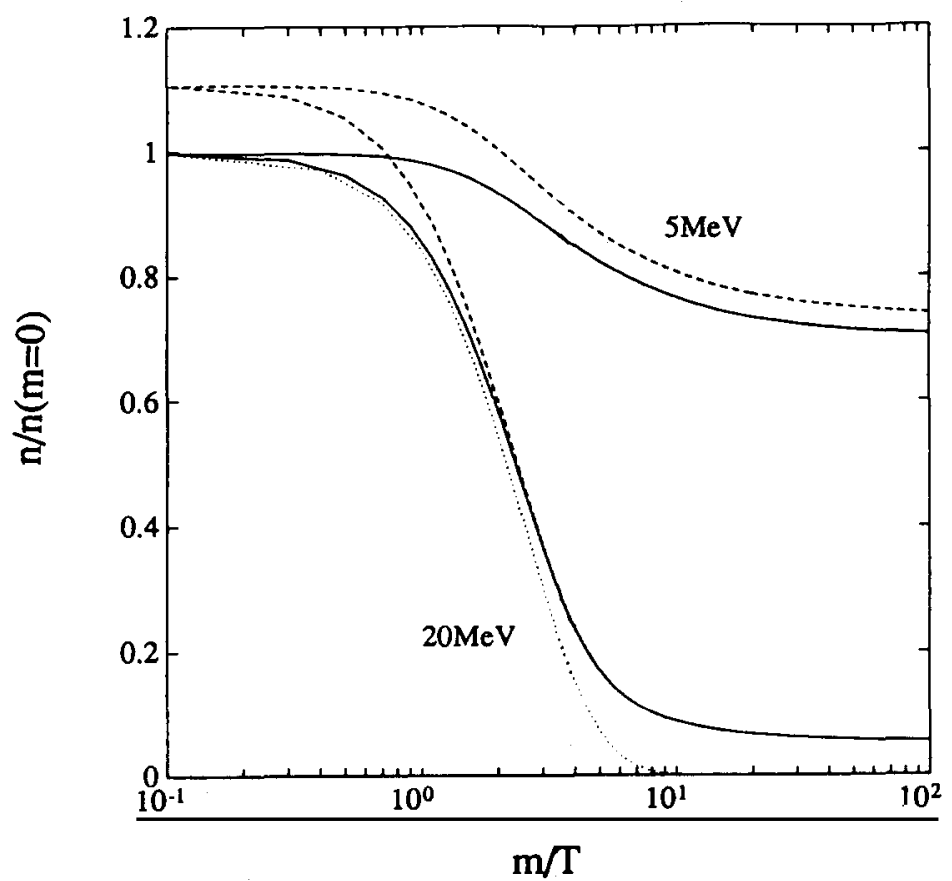

Fig. 1. The ratio of the number density of a particle of mass $m=5 \mathrm{MeV}$ and $m=20 \mathrm{MeV}$ to the number density of massless particles $n / n(m=0)$ as a function of the temperature $T$. Solid lines correspond to the FD-statistics and the dashed lines to the MB-statistics. The dotted line shows the equilibrium number density. 
where $V=\sqrt{1-4 m^{2} / s}$ and $s=T^{2}\left(p^{2}-q^{2}\right)=m^{2}\left(p^{2}-q^{2}\right) / x^{2}$. The corresponding equation in the MB-limit is

$$
\frac{\mathrm{d} z}{\mathrm{~d} x}=-\frac{K_{1}(x)}{K_{2}(x)}-6.15 \times 10^{-4} \alpha\left(\frac{m}{\mathrm{MeV}}\right)^{3} \frac{K_{1}^{2}(x)}{K_{2}(x)} \sinh z
$$

We have solved numerically eqs. (25) and (27). In fig. 1 we show our results for the evolution of the number density. In all cases we have used the value $\alpha=20$ for the interaction strength. Fig. 1 shows that if the decoupling from equilibrium occurs at $T \leqq \mathrm{O}(\mathrm{m})$, then the MB-approximation overestimates the true value of the quantity in question, the effect being the largest in the region $T \gg m$. In the last case however one can simply find the frozen number density of particles in question without solving the kinetic equation noting that it is equal to the equilibrium number density of massless particles at the moment of decoupling. In fig. 2 we show the asymptotic value (for $T \simeq 0$ ) of the scaled number density as a function of mass. We also show the curves corresponding to an approximate analytic solution of eq. (1) $[9,15]$,

$$
f(0) \equiv \frac{n}{s} \simeq \frac{H\left(T_{\mathrm{f}}\right)}{s_{\mathrm{f}}\left\langle v_{\mathrm{M} \phi 1} \sigma\right\rangle_{\mathrm{f}}}
$$

Here the index f refers to the freeze-out temperature, which is calculated from the equation (assuming $\left\langle v_{\mathrm{M} \varnothing 1} \sigma\right\rangle=$ const.)

$$
\frac{m}{T_{\mathrm{f}}} \simeq \ln C-\frac{1}{2} \ln \ln C
$$

where $C$ is given by

$$
C \equiv\left(\frac{4}{45} \pi^{3} g(T)\right)^{-1 / 2} \frac{g}{(2 \pi)^{3 / 2}} \delta(\delta+2) \alpha G_{\mathrm{F}}^{2} M_{\mathrm{P} 1} \frac{m^{3}}{32 \pi}
$$

For the free parameter $\delta$, we have used the commonly used value $\delta(\delta+1)=1$ $[2,9,15]$. Here one can see how the correction due to the FD-statistics dies away asymptotically as the mass increases. This reflects the fact that, while also in this region the MB-solution is initially inadequate, the particle ensemble remains in equilibrium long enough for the asymptotics to be well described by the MB-statistics. One should note also that expression (28) gives a very poor approximation in the region of $m \leqslant T_{\mathrm{d}}$. In particular when one is concerned with very accurately known quantities such as restrictions derived from the nucleosynthesis considerations, one should be careful to use at least the accurate numerical solution of eq. 


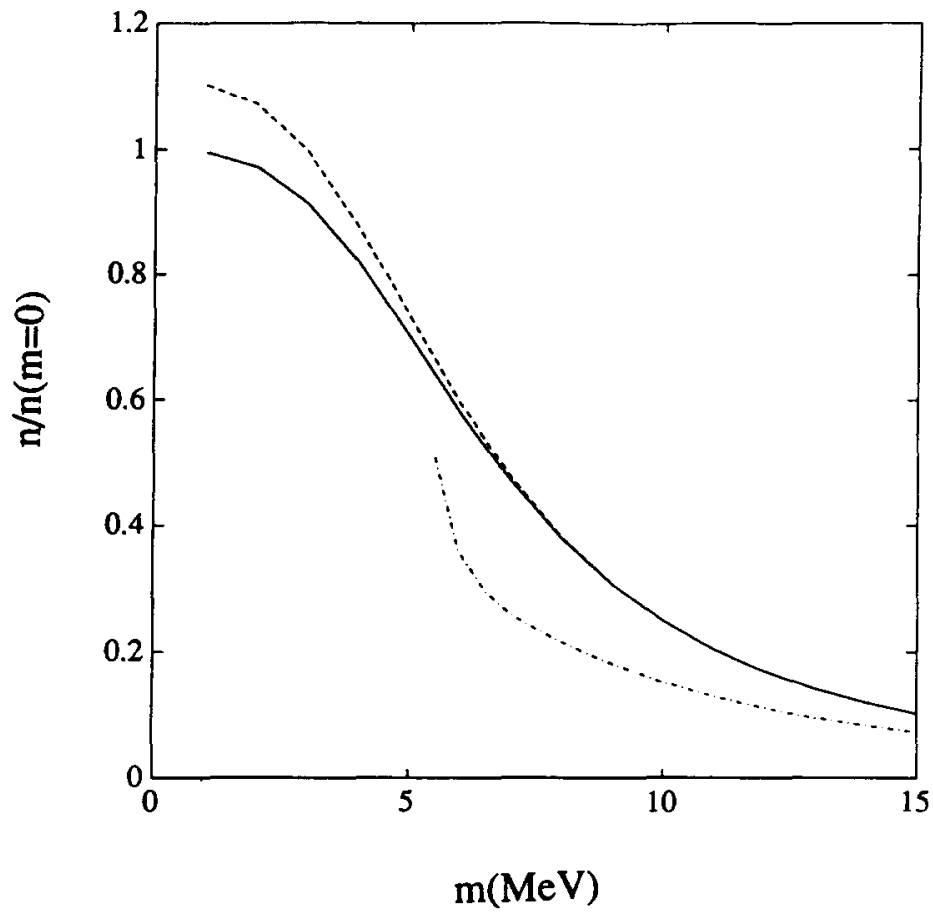

Fig. 2. The asymptotic limit $(T=0)$ of the scaled number density $n / n(m=0)$ as a function of the neutrino mass. The solid line corresponds to the FD-statistics, the dashed line to the MB-statistics and the dash-dotted line to the approximation (28).

(1) and in some cases the numerical solution of eq. (15) with the correct statistics.

\section{Conclusions and discussion}

We have derived an evolution equation for the pseudo-chemical potential $z(T)$ in the early universe, that allows one to obtain various thermodynamical quantities as functions of the temperature, as simple integrals over momentum, with the correct Fermi-Dirac statistics. We have also obtained the reduction of this equation in the usual Maxwell-Boltzmann approximation and compared this limiting case of our result with the literature. We have solved the evolution equations numerically for a simple toy model, where the interaction matrix element was assumed to be a constant. Our results confirm the expectation that the FD-corrections are sizable in the region, where the decoupling from the thermal equilibrium occurs at temperatures of the order of, or less than the neutrino mass. One should especially note that the use of some popular analytic approximations for the relic number density in this region is very ill advised. 
The use of correct statistics could be important, for example in the case of a few $\mathrm{MeV}$ tau neutrino, which clearly would decouple while semirelativistic. It has been noted elsewhere [10] that such particles would strongly influence the nucleosynthesis due to their potentially large energy density, which would tend to increase the expansion rate of the universe. In these studies the MB-approximation was used. As follows from our results the correct statistics gives 5-10\% smaller results and correspondingly weaker bounds on the tau neutrino mass.

Let us finally mention that we have assumed here that both helicity states are equally populated. It might not be true for the chiral interactions. This is especially the case for the standard model interactions and therefore for the aforementioned tau neutrino. This problem, together with the calculation of Fermi corrections to the realistic case of tau-neutrino freezing will be pursued elsewhere [12].

A.D. wishes to thank the Center for Particle Astrophysics at UC Berkeley, where this work started, and the Physics Department of the University of Michigan, where it ws completed, for the hospitality. K.K. wishes to thank the Research Institute for Theoretical Physics, University of Helsinki, where part of this work was done, for hospitality and the Emil Aaltonen foundation and the Finnish Academy for financial support. This research is supported by DOE grant DEAC02-83ER40105.

\section{References}

[1] M.I. Vysotsky, A.D. Dolgov and Ya.B. Zeldovich, JETP Lett. 26 (1977) 188;

B.W. Lee and S. Weinberg, Phys. Rev. Lett. 39 (1977) 165

[2] G. Steigman, Ann. Rev. Nucl. Part. Sci. 29 (1979) 313;

R.J. Scherrer and M.S. Turner, Phys. Rev. D33 (1986) 1585

[3] K. Griest and D. Seckel, Nucl. Phys. B283 (1987) 681

[4] K. Enqvist, K. Kainulainen and J. Maalampi, Nucl. Phys. B316 (1988) 456

[5] D.E. Brown and L.J. Hall, Phys. Rev. D41 (1990) 1067;

see also E.W. Kolb and M.S. Turner, The early universe (Addison-Wesley, Redwood city, CA, 1990), and references therein

[6] Ya.B. Zeldovich, L.B. Okun and S.B. Pikelner, Sov. Phys. Uspekhi 8 (1965) 702

[7] J. Bernstein, L.S. Brown and G. Feinberg, Phys. Rev. D32 (1992) 3261

[8] M. Srednicki, R. Watkins and K.A. Olive, Nucl. Phys. B310 (1988) 693

[9] P. Gondolo and G. Gelmini, Nucl. Phys. B360 (1992) 145

[10] E.W. Kolb et al., Phys. Rev. Lett. 29 (1991) 533

[11] A.D. Dolgov Sov. J. Nucl. Phys. 32 (1980) 831

[12] A.D. Dolgov and K. Kainulainen, in preparation

[13] S.R. de Groot, W.A. van Leeuwen and Ch.G. Weert, Relativistic kinetic theory (North-Holland, Amsterdam, 1980)

[14] L.D. Landau and E.M. Lifshitz, The classical theory of fields (Pergamon, Oxford 1975)

[15] E.W. Kolb and M.S. Turner, The early universe (Addison-Wesley, Redwood city, CA, 1990) 